\title{
Food for thought from plant and animal genomes
}

\author{
Emily Abrash*
}

\section{Abstract \\ A report on the Plant and Animal Genome XXI meeting, held in San Diego, USA, January 12-16, 2013.}

On 12 January, on a morning full of blue sky and cold sunshine, the Plant and Animal Genome XXI meeting opened its doors for the 21st time at the Town and Country Hotel in San Diego. I arrived a couple of hours late, a newbie toting a roller suitcase, a little unprepared for the sheer scope of the meeting I was about to attend. The diversity of topics and attendees at the meeting, "The Largest Ag-Genomics Meeting in the World', was stunning. Within my first hour, I would wind up eating my boxed lunch with a member of the transitional government of Egypt, who moonlights as a grad student in Colorado; and within my first afternoon, I would hear talks about drought resistance in rice, marker-assisted breeding in sweet cherry and transgressive segregation in cotton, not to mention the 12 concurrent sessions on topics from citrus genomes to swine breeding. As a plant developmental biologist interested in international agriculture, I chose talks with an eye towards the border between basic and applied research, the brackish zone where molecular biological innovations find their way into research centers, field trials, and ultimately, farmers' fields. In this report, I will present examples of the diverse and exciting work being done at this intersection, and will conclude by highlighting some emerging trends and challenges on the horizon.

\section{Basic research with agricultural relevance}

Some work presented at the meeting was not directly translational, but rather examined basic biological questions using a system or angle relevant to agriculture. Steve Jacobsen's (University of California, Los Angeles and Howard Hughes Medical Institute, USA) plenary talk provided an excellent example of such work. Jacobsen, who has done extensive research on the epigenetics of

${ }^{*}$ Correspondence: eabrash@stanford.edu

Stanford University Department of Biology, 371 Serra Mall, Stanford, CA 94305, USA
Arabidopsis thaliana, gave a talk in an earlier session on the genome-wide DNA methylation analysis of a panel of 86 Arabidopsis silencing mutants. As part of his plenary presentation, however, he examined how similar techniques can be used to analyze the epigenetic changes that occur during rice callus transformation. By profiling DNA methylation patterns of individual callus-derived lines, Jacobsen's group showed that regenerants lose methylation relative to parental plants, and that loss typically occurs in certain regions of the genome (although the exact pattern differs among regenerated plants). As production of transgenic cereals generally requires a callus stage, this work will be valuable in understanding variability in crop transformation outcomes.

Another example of elegant basic research with agricultural significance was Sue Wessler's (University of California, Riverside, USA) work on transposable elements in rice. Her work focuses on mPing, an element currently undergoing a burst of amplification in several rice strains. By monitoring movement of mPing in individual plants over multiple generations, Wessler's group has been able to demonstrate that a non-coding insertional preference, together with the ability to confer adaptive changes in gene expression (for example, stress inducibility), may explain why mPing amplification is tolerated. Understanding how transposable elements such as mPing interact with and influence their hosts provides insight into an important force shaping evolution of crop genomes.

\section{Functional molecular genetics in crop species}

Other work presented at the meeting used sophisticated molecular-genetic approaches to directly address practical agricultural questions. One beautiful example came from Rico Gamuyao's (International Rice Research Institute, Philippines) analysis of the Pup1 quantitative trait locus (QTL) for elevated phosphorus uptake, which was originally derived from the Indian rice cultivar Kasalath, which is adapted to nutrient-poor soil. Pup1 was mapped to a small chromosomal insertion absent from the Nipponbare reference genome. A protein kinase gene within the insertion was named phosphorus-starvation tolerance 1 (Pstol1), and its function was confirmed by overexpression of the gene, which conferred increased yields and greater phosphorus accumulation under phosphorus-deficient conditions. The basis of this tolerance 
appears to be developmental: Pstol1 overexpressors have enhanced root growth, while Pstol1 RNA interference plants have reduced root growth and fewer crown roots. The Pup1 locus has been incorporated into breeding programs and field trials, such that this elegant study also provides a practical resource for crop improvement.

Another example of molecular-genetic research with direct benefits for crop breeding comes from Yusaku Uga's (National Institute of Agrobiological Sciences, Japan) work on drought resistance in rice. Uga and colleagues identified, mapped and cloned an upland ricederived QTL called deeper rooting 1 (Dro1), which increases depth of rooting and thus improves drought tolerance. Dro1 encodes an auxin-regulated protein of unknown function expressed in dividing cells of the root tip and appears to promote deeper rooting by enhancing asymmetric growth in response to gravity. Uga has also identified QTLs controlling root thickness, length and volume, and these are currently being mapped and cloned, as well as pyramided with Dro1 to enhance drought tolerance.

\section{Emerging model systems for crops and biofuels}

While many attendees were working directly on crops, the meeting was also a gathering place for those studying emerging models related to agriculturally important species. Brachypodium distachyon, a C3 grass related to barley and wheat, is an emerging model for temperate grasses, while Setaria viridis, a C4 grass related to maize, switchgrass and foxtail millet, is a closer model for tropical grasses. This year, presentations incorporating $B$. distachyon examined traits as diverse as cell wall composition (Sam Hazen, University of Massachusetts Amherst, USA), iron homeostasis (Elsbeth Walker, University of Massachusetts Amherst, USA) and arbuscular mycorrhizal symbiosis (Liudmila Yafremava, University of Illinois, USA). For the newer model S. viridis, Tom Brutnell (The Donald Danforth Plant Science Center, USA) described exciting progress in development of resources, including Agrobacterium-mediated transformation with 7 to $10 \%$ efficiency and generation of a mutant population to screen for $\mathrm{C} 4$ photosynthetic defects. As these models mature, they will have the potential to accelerate functional studies in grasses and yield findings that can readily be translated to crop and biofuel species.

\section{Genomic selection and genome-wide association studies in crop breeding}

Alongside conventional mapping and molecular-genetic approaches, a major theme at this year's Plant and Animal Genome meeting was the potential of genomic selection and genome-wide association studies (GWAS) to facilitate gene discovery and accelerate the breeding cycle in crops. While broadly used in human genetics, these approaches are still being explored and developed for crop species and livestock; as discussed by Rex Bernardo (University of Minnesota, USA) and David Habier (Iowa State University, USA), a diverse array of factors, including properties of populations under study, the heritability of the trait of interest, and the type of genetic interactions involved, affect both the predictive capabilities of genomic selection and GWAS and the type of strategy best applied.

Nonetheless, reports on the incorporation of largescale genomic strategies into public breeding efforts illustrate their clear and growing utility. For example, Susanne Dreisigacker (CIMMYT, Mexico) described the progression of her institute's wheat breeding program from a phenotyping-based approach to marker-assisted selection and, ultimately, to genomic selection using high-throughput sequencing. Similarly encouraging results in sorghum were presented by Geoffrey Morris (University of South Carolina, USA), who conducted GWAS for height and inflorescence architecture as part of a collaboration with ICRISAT centers. This work included 971 diverse sorghum accessions, demonstrating the power of GWAS to identify functionally relevant variation within large germplasm collections. Comparable strategies are also being used to exploit maize genetic diversity, as highlighted in Edward Buckler's (USDA-ARS, USA) work combining maize HapMap data, QTL analysis of nested association mapping lines, and genotyping-by-sequencing (reduced representation genotyping) to perform GWAS for developmental and metabolic traits. Next-generation sequencing has made genotyping more cost-effective than phenotyping in many cases, such that genomic selection and GWAS may allow both faster and more economical crop improvement.

\section{Future directions and challenges}

To attend the Plant and Animal Genome meeting is to come away inspired, but also wanting more: the next paradigm-shifting discovery, the next page-turn of the story. What, then, are some emerging trends and challenges? Certainly, integration of genomic selection and GWAS approaches into breeding programs will be a major project of the coming years. Another emerging paradigm of interest is automated large-scale phenotyping (as in the Adelaide 'Plant Accelerator' facility described by Mark Tester, The University of Adelaide, Australia). Yet I would suggest one of the most exciting challenges is that presented by Jeffrey Bennetzen (University of Georgia, USA) in his excellent talk on crop diversification: that of how new technologies and conventional approaches can best be used to improve underutilized staple crops from around the world. As Bennetzen discussed, species important in developing countries, such 
as sorghum, millet and tef, have potentially valuable properties such as enhanced stress tolerance. Yet these 'orphan crops' are little used in the developed world and, correspondingly, under-improved, such that conventional breeding might yield superior varieties for a relatively small investment. Even simple monogenic mutations could markedly improve such crops: fonio millet displays grain shattering, while tef suffers from lodging (collapsed stems), liabilities that could be eliminated by loss-offunction mutations conferring shatterproof grain and dwarfism, respectively.

Bennetzen's group is following up on these particular examples with targeting induced local lesions in genomes (TILLING), and it will be exciting to see which 'silver bullet' mutations are recovered. But there are undoubtedly other crops for which a similarly modest investment in conventional breeding, mutagenesis or genomic selection would yield a dramatic payoff in terms of crop improvement. Such an effort would not only provide superior lines for some of the world's most resource-poor farmers, but might also, as Bennetzen suggests, produce stress-tolerant crops of worldwide benefit in a changing climate.

\section{Acknowledgements}

I apologize that descriptions of the many other outstanding presentations cannot all be included due to space constraints.

\section{Abbreviations}

GWAS, genome-wide association study; QTL, quantitative trait loci.

\section{Competing interests}

The author declares that she has no competing interests.

Published: 27 February 2013

doi:10.1186/gb-2013-14-2-302

Cite this article as: Abrash E: Food for thought from plant and animal genomes. Genome Biology 2013, 14:302. 